\title{
Liver resection following FOLFOXIRI plus bevacizumab: a detailed pathological review
}

\author{
H Walter ${ }^{1}$ and A L Thomas ${ }^{\star}, 1$ \\ ${ }^{1}$ Department of Cancer Studies and Molecular Medicine, University of Leicester, Leicester LE15WW, UK
}

Currently, 'the pipeline' of drugs in development for the treatment of colorectal cancer is limited. As such, it is essential that we understand and maximise the therapeutic potential of our available drugs. A good example of this has been the development of triplet regimens with 5FU/LV or capecitabine, irinotecan and oxaliplatin (FOLFOXIRI), and, latterly, the addition of bevacizumab to FOLFOXIRI. A phase II study in patients with advanced colorectal cancer receiving first-line treatment with FOLFOXIRI-BEV achieved an impressive median progression-free survival (PFS) of 13.1 months (the first time PFS went beyond the 1-year bar) with a response rate of $77 \%$, and $32 \%$ of patients proceeded to surgical resection of metastases (Masi et al, 2010). The hotly awaited phase III TRIBE trial conducted at 35 Italian centres and reported at the 2013 Gastrointestinal Cancers Symposium achieved its objective of confirming the superiority of FOLFOXIRI $v s$ FOLFIRI in combination with bevacizumab in terms of PFS (12.2 vs 9.7 months) (Loupakis et al, 2013a). Data regarding overall survival have not yet reached maturity.

So how do we select the most appropriate group of patients for this intensive regimen? Biomarkers that aid the personalisation of chemotherapy strategies are desperately needed. There is no doubt that some patients respond very well to anti-angiogenic approaches, but these effects are diluted out by the non-responders - a predictive biomarker is overdue, particularly in relation to bevacizumab. What does the paper by Loupakis et al (2013b) in this issue of BJC add to our knowledge base? Certainly it is timely, with the current interest around triplet + regimens in advanced colorectal cancer.

In summary, Loupakis et al (2013b) is a detailed pathological study looking at the role of an intensive first-line regimen with bevacizumab in patients with colorectal liver metastases (CLM). Pertinent questions such as complete pathological response (cPR), tumour regression grade (TRG), tumour necrosis and fibrosis, and those on treatment-related hepatic toxicities, are analysed. Pathological response in terms of viable tumour cells and histopathological regression grade has been shown to correlate with improvements in clinical outcome. In this study, with the use of bevacizumab in combination with triplet regimens, a cPR rate of $16 \%$ was documented, with $11 \%$ in the chemotherapy-alone group. While this difference is not statistically significant, the addition of bevacizumab to FOLFOXIRI increased the chances of achieving a TRG of 1, 2 or 3 (odds ratio 1.833) and increased the degree of necrosis.

This issue of necrosis is interesting. To date the actual mechanism by which inhibition of the VEGF/VEGFR axis by bevacizumab interferes with tumour growth remains elusive (Ellis and Hicklin, 2008). How important and credible is the observation of the increased necrosis? Is this suggesting that we should revisit the 'vaso-constriction' mechanism of action? Using both dynamic contrast-enhanced MRI and PET imaging, it is possible to detect necrosis in tumours and indeed quantify it (Morgan et al, 2006). Furthermore, these studies can be performed early in the treatment of patients, potentially allowing them to stop such a clinically toxic regimen if no change in necrosis is seen and equally to determine how many cycles should be given pre-operatively. Should we be reviewing these imaging studies to try and develop predictive biomarkers measuring necrosis?

The information from surgical resection specimens will only have limited clinical utility. One important application would be to design a study randomising patients post liver resection, with the stratification based on these pathological parameters, to post surgical chemotherapy or not. This would answer an important clinical question. Certainly this detailed pathological analysis requires validation and quality assurance, and it is questionable whether many pathological departments could deliver this information as a matter of routine.

The second important question addressed in this study was the risk of liver toxicity. Is the use of intensive therapies and associated increase in response rate outweighed by the risk of increased liver toxicity in a potentially curative setting? It appears not. No statistically significant difference was seen regarding sinusoidal dilatation, parenchymal steatosis, steatohepatitis and parenchymal necrosis between the chemotherapy-alone and bevacizumab groups. Aside from parenchymal necrosis, chemotherapy-naive patients also exhibited such toxicities, albeit to a lesser degree.

This study is limited by its retrospective, non-randomised and exploratory nature. Data on overall survival are immature, but despite this, findings demonstrate that an intensive approach (with or without bevacizumab) can produce high pathologic response rates. The addition of bevacizumab to FOLFOXIRI appeared to further improve the pathologic response and degree of necrosis of 
CLM in this analysis without significant liver toxicity. This is very reassuring, particularly for the hepatobiliary surgeons. However, it still does not help us select the most suitable patient group for this aggressive approach. The necrosis data are interesting. There is a clear need to continue this work in prospective studies, and we would recommend correlating the results with concurrent imaging studies measuring enhancement changes.

\section{REFERENCES}

Ellis LM, Hicklin DJ (2008) VEGF-targeted therapy: mechanisms of a nti-tumour activity. Nat Rev Can 8: 579-591.

Loupakis F, Cremolini C, Masi G, Lonardi S, Zagonel V, Trenta P, Tomasello G, Ronzoni M, Ciuffreda L, Zaniboni A, Tonini G, Buonadonna A, Valsuani C, Chiara S, Carlomagno C, Boni C, Marcucci L, Boni L, Falcone A (2013a)
FOLFOXIRI plus bevacizumab (bev) versus FOLFIRI plus bev as first-line treatment of metastatic colorectal cancer (MCRC): results of the phase III randomized TRIBE trial. J Clin Oncol 31(Suppl 336): abstract 1.

Loupakis F, Schirripa M, Caparello C, Funel N, Pollina L, Vasile E, Cremolini C, Salvatore L, Morvillo M, Antoniotti C, Marmorino F, Masi G, Falcone A (2013b) Histopathologic evaluation of liver metastases from colorectal cancer in patients treated with FOLFOXIRI plus bevacizumab. Br J Cancer 108(12): 2549-2556.

Masi G, Loupakis F, Salvatore L, Fornaro L, Cremolini C, Cupini S, Ciarlo A, Del Monte F, Cotesi E, Amoroso D, Granetto C, Fontanini G, Sensi E, Lupi C, Andreuccetti M, Falcone A (2010) Bevacizumab with FOLFOXIRI (irinotecan, oxaliplatin, fluorouracil, and folinate) as first-line treatment for metastatic colorectal cancer: a phase 2 trial. Lancet Oncol 11: 845-852.

Morgan B, Utting J, Higginson A, Thomas AL, Steward WP, Horsfield MA (2006) A simple, reproducible method for monitoring the treatment of tumours using dynamic contrast enhanced MRI. Br J Cancer 94: $1420-1427$. 